\title{
DIELECTRIC BEHAVIOR OF NANO BARIUM TITANATE FILLED POLYMERIC COMPOSITES
}

\author{
A. PRATAP* \\ Applied Physics Department, Faculty of Technology and Engineering \\ The Maharaja Sayajirao University of Baroda, Vadodara - 390001, India \\ E-mail: apratapmsu@yahoo.com
}

\section{N. J. JOSHI, P. B. RAKSHIT, G. S. GREWAL, V. SHRINET}

Electrical Research \& Development Association ERDA Road, Makarpura, Vadodara - 390 010, India nirav.joshi1986@gmail.com

\begin{abstract}
Rapid growth of electronic industry requires development of new materials that combine the high dielectric constant intrinsic to ferroelectric ceramic materials with easy processing characteristics of polymers. Ceramic-polymeric composites possess interesting properties for a variety of electronic applications including passive electronic devices. In fact, polymer-ceramic materials have drawn lot attention for use in microelectronic packaging, because they can give higher performance with lower cost, size and weight.

In this work, attempts are made to prepare ceramic polymer composites followed by characterization of dielectric properties. The Barium Titanate ceramic powders are synthesized using the hydrothermal process. Crystal structure and crystallite size of particles are determined using X-ray diffraction. Silane treatment is carried out on Barium Titanate powder to increase its compatibility with polymer, followed with preparation of ceramic polymer composites. Epoxy and polyvinyledene fluoride (PVDF) polymers are used as matrices for preparation of the composites. The proportion of nanopowder is varied from 60 to $90 \mathrm{wt} \%$. Dielectric properties such as volume resistivity, dielectric constant, dissipation factor are evaluated. Results indicate that the dielectric constant and dissipation factor vary between $18-140$ and 0.01 to 0.09 , respectively as the relative ratio of polymer and silane modified Barium Titanate is varied. Specifically, at $90 \mathrm{wt} \%$ of $0.1 \mathrm{wt} \%$ silane modified Barium Titanate, the highest dielectric constant of 140 along with dissipation factor of 0.07 is obtained in the epoxy based system.
\end{abstract}

Keywords: Polymer-Barium Titanate Composites, Dielectric Properties, Surface Modification, Epoxy, PVDF

\section{Introduction}

Polymeric composite materials, made of epoxy resin and dielectric ceramic particles, have been reported to posses interesting properties for a variety of electronic applications, such as passive electronic devices. Polymer-ceramic materials have aroused a lot of attention especially for uses in microelectronic packaging, because they can have high performances with lower cost, size and weight ${ }^{1}$. Composite dielectric behavior can be 
affected by the presence of porosity and filler distribution. Besides the necessary amount of filler has to be enough to obtain a rather high composite permittivity. However, the processing step is more difficult when high filler amounts are incorporated ${ }^{2}$. Sometimes solvents are added during the mixing of filler with polymer for easy processing and mixing to enable enhancements to the distribution of filler in the polymer matrix. The diluted resin allows the incorporation of higher filler concentrations without significantly increasing the viscosity. Ceramic-polymer composites can typically be fabricated using low temperature processing techniques ${ }^{3-8}$. The traditional approach for preparation of such composites, especially composite films, is to mix a dielectric polymer solution and submicron- or micron-sized ferroelectric particulates, and evaporate the solvent of the polymer solution ${ }^{3-8}$. The most common process for enhancing the dielectric permittivity of a polymer is to disperse a high dielectric permittivity insulating ceramic powder such as Barium Titanate $\left(\mathrm{BaTiO}_{3}\right)^{9-11}$ or lead titanate $\left(\mathrm{PbTiO}_{3}\right)^{12}$ into the polymer to form a composite. In order to obtain a high value of dielectric permittivity, large amount of fillers has to be loaded, resulting in loss of flexibility and inhomogeneous composites. Another approach to obtain a high dielectric permittivity composite is to utilize a conductive filler (near its percolation threshold) to produce the composites. In this work, attempts have been made to prepare polymer-Barium Titanate composites and their dielectric properties are studied for determining similarity for a variety of dielectric applications, such as in dielectric capacitors ${ }^{13}$, $\operatorname{transducers}^{14}$ and tunable phase shifters ${ }^{15}$.

An interesting feature of nanometric sized Barium Titanate is the fact that when the particle size is greater than about $10 \mathrm{~nm}$, the dielectric behavior is ferroelectric-with a permanent frozen in polarization. However, when the particle size approaches size scale of about $1 \mathrm{~nm}$, the dielectric behavior switches over to paraelectric behavior, with field dependent polarization. Paraelectric Barium Titanate tends to have very low dissipation factor and hence is ideally suitable for AC capacitors ${ }^{13}$.

Many researchers have conducted chemical surface modification of $\mathrm{BaTiO}_{3}$ nanoparticles in order to improve its compatibility with the polymer matrix and to improve the dielectric properties ${ }^{16-17}$. Among various surface modification processes, the use of silane coupling agents is the most-accepted method, because various functional groups can be introduced on the particle surface. Dang et al. ${ }^{16}$ have modified the $\mathrm{BaTiO}_{3}$ surface with aminopropyltriethoxysilane to prepare $\mathrm{BaTiO}_{3} /$ poylvinyledene fluoride (PVDF) composites. It has been reported that the compatibility between $\mathrm{BaTiO}_{3}$ surface and PVDF, and the composite's dielectric properties, was increased by modifying the $\mathrm{BaTiO}_{3}$ surface with $1 \mathrm{wt} \%$ of aminopropyltriethoxysilane. However, the effect of surface structure on the dielectric property of nanocomposite materials is still not well understood. Studies have also been carried out on the effect of solvent on the surface structure of Barium Titanate after its modification by silane coupling agents.

The silane modified $\mathrm{BaTiO}_{3}$ nanopowder is mixed with epoxy and PVDF to prepare polymer-ceramic composites, which is subsequently evaluated for its dielectric properties. 


\section{Experimental}

Barium Titanate nanopowder was synthesized using the hydrothermal process ${ }^{18}$. Glycidoxymethoxy silane was applied onto ceramic particles as diluted aqueous solutions of $0.1,0.3$ and $0.5 \mathrm{wt} \%$ (as a percentage of Barium Titanate content) of silane in order to improve processability and increase filler wet-out and dispersion. Aqueous solution was prepared, adjusting the $\mathrm{pH}$ of the water to 3.5 with acetic acid and then introducing the silane. After the silane was added to the acidified water, the system was stirred for about $15 \mathrm{~min}$ before it hydrolyzed and formed a clear and homogeneous solution. The fillers were mixed with the silane for several minutes without additional solvent. After applying the silane, the $\mathrm{BaTiO}_{3}$ was dried at $110{ }^{\circ} \mathrm{C}$ to avoid condensation of silanol groups at the surface. The detection of small quantities of silane in the products after the silanisation process was achieved by Fourier Transform Infrared Spectrometry (FTIR). Weighed amount of silane modified Barium Titanate powder and epoxy resin were mixed efficiently using mechanical stirrer for $5 \mathrm{~min}$ at speed of 1500 RPM. Five wt\% of Xylene (as percentage of epoxy resin) was added to the mixture to reduce the viscosity of the resin. The mixture was stirred and a known amount of commercially available curing agent was added in the reaction mixture and stirred for five more seconds. The whole reaction mixture was subsequently poured in the mould. The material was cured at room temperature for four hours followed by post curing at $60{ }^{\circ} \mathrm{C}$ for one hour. The composite so prepared was released out of mould and used for evaluation of the dielectric properties. Similarly, PVDF-Barium Titanate composites were also evaluated for the dielectric properties.

\section{Characterization}

The crystallite size and crystal structure of Barium Titanate was measured by linebroadening measurements using X-ray diffraction on a Bruker AXS D8 Advance X-Ray diffractometer using $\mathrm{Ni}$ filtered $\mathrm{Cu}-\mathrm{K} \alpha$ radiation. Normal XRD scans with step resolution of $0.02^{\circ}$ with time step of $0.5 \mathrm{sec}$ were used. To ensure stability of the measurements with respect to change in resolution in angular coordinates and time, measurements were repeated with angular step size (in $2 \theta$ ) of $0.05^{\circ}$ with time step of $2 \mathrm{~s}$. The $\mathrm{Cu}-\mathrm{K} \alpha 2$ diffraction signal was removed by a standard stripping procedure to obtain the correct lattice parameters and grain size. The confirmation of incorporated functional groups was carried out using Fourier Transform Infrared spectroscopy (FTIR). The samples for IR analysis were prepared as potassium bromide pellets. The scans were obtained using Perkin Elmer Spectrum BX Fourier transform infrared (FTIR) system (Huenenberg Switzerland). Dielectric measurements were performed using a Model 1658, RLC Bridge in the frequency range from $20 \mathrm{~Hz}$ to $100 \mathrm{kHz}$. Temperature was varied from room temperature to $150{ }^{\circ} \mathrm{C}$. 


\section{Design of Experiments}

The composites were prepared using epoxy and PVDF polymer matrices and Barium Titanate was increased from 60 to $90 \mathrm{wt} \%$. Initially, the $90 \mathrm{wt} \%$ silane $(0.1,0.3$ and 0.5 wt \%) modified Barium Titanate was mixed with the epoxy resin and the samples were cured using the cross linking agent. These samples were characterized and the dielectric properties studied. It was found that $90 \mathrm{wt} \%$ of $0.1 \mathrm{wt} \%$ silane modified Barium Titanate exhibited good results and on increasing the silane concentration, the properties deteriorated. Low silane concentration modified interfaces thus helped in retaining the electrical properties of the composite. However, higher silane concentrations increased the porosity and defects, and impoverished the dielectric properties. Therefore, further work was done using the $0.1 \mathrm{wt} \%$ silane modified powder only.

\section{Results and Discussion}

\subsection{X-Ray diffraction studies}

The crystallite size and crystal structure was measured by line-broadening measurements using X-ray diffraction. Figure 1 presents the XRD spectra of sintered Barium Titanate pellet synthesized by the hydrothermal process. Based on this plot, the $\mathrm{BaTiO}_{3}$ cubic phase is characterized as having a cell parameter of $3.999 \AA$ with space group Pm-3m (221). This phase completely conforms to $\mathrm{BaTiO}_{3}$ phase as per standard XRD JCPDS card 01-074-1962. The average crystallite size of the phase is $\sim 33 \mathrm{~nm}$ (using Scherer equation), confirming the nanoscale nature of the sintered material.

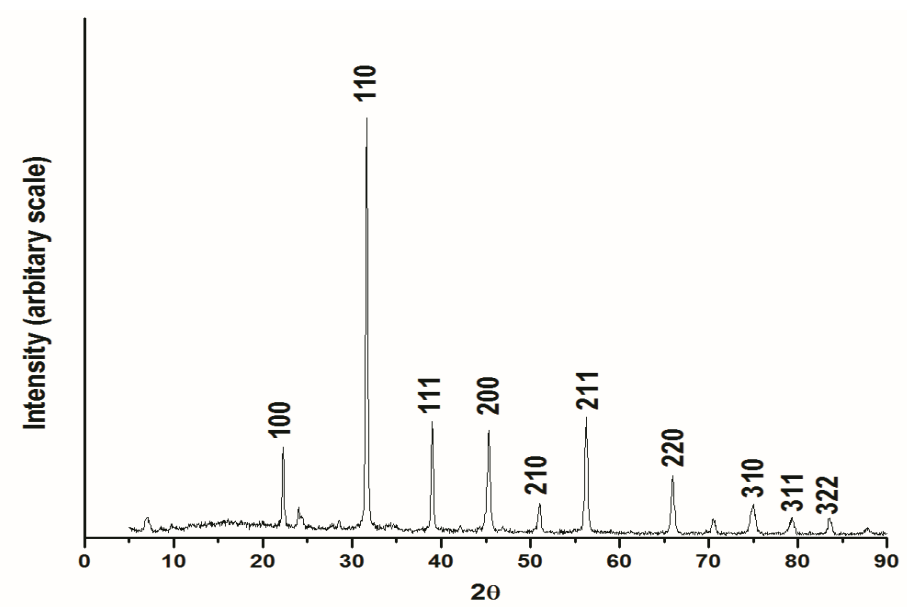

Fig. 1. XRD Plot of sintered Barium Titanate prepared by hydrothermal process.

\subsection{FTIR analysis}

Figure 2 (a-b) shows the FTIR spectra of the Barium Titanate powder and the powder modified with $0.1 \mathrm{wt} \%$ of silane. The broad band at 568 to $577 \mathrm{~cm}-1$ are due to Ti-O 
stretching modes of $\mathrm{BaTiO}_{3}{ }^{19-20}$, while peaks at 1430 and $1625 \mathrm{~cm}-1$ are related to the $\mathrm{CO}_{2}$ absorption ${ }^{20}$. Moreover, peaks at 853 are the characteristic peak of $\mathrm{C}-\mathrm{C}$ stretching modes. Figure 3 a shows an additional peak at $1364 \mathrm{~cm}-1$ corresponding to organic groups of silane $\mathrm{e}^{21-22}$.

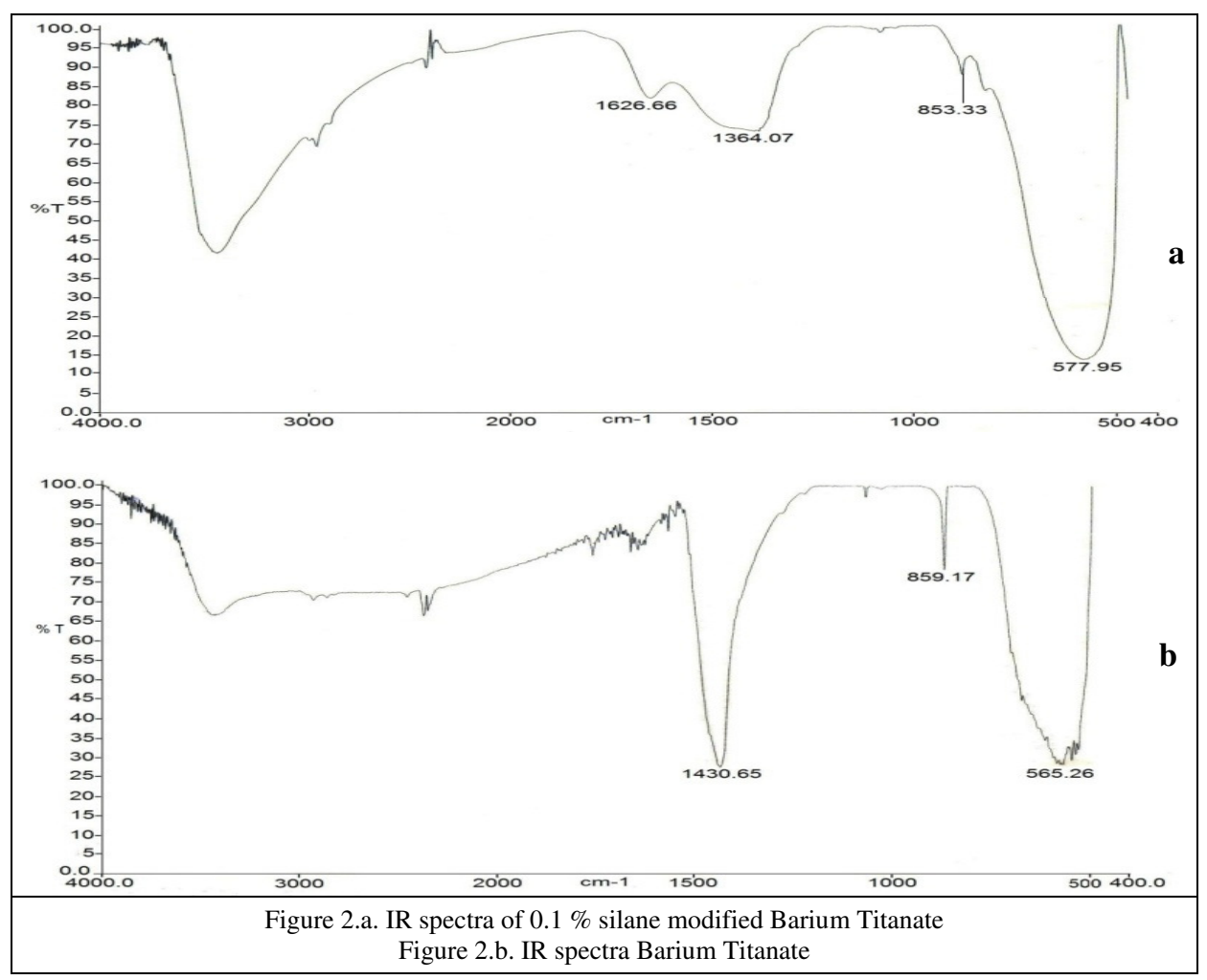

\subsection{Dielectric properties of ceramic-polymer composites}

Figure 3 (a-b) presents plots of dielectric constant versus concentration of Barium Titanate in epoxy and PVDF polymer matrices respectively. It is clearly observed that as concentration of Barium Titanate is increased from 60 to $90 \mathrm{wt} \%$ with respect to the polymer, the dielectric constant increases and $90 \mathrm{wt} \%$ shows the maximum dielectric constant for each of the two composites systems. The highest dielectric constant for $\mathrm{BaTiO}_{3}$-epoxy is observed to be 110 whereas for the silane modified $\mathrm{BaTiO}_{3}$ - epoxy it is observed as 140 . Similarly for the $\mathrm{BaTiO}_{3}-\mathrm{PVDF}$ composites the highest dielectric constant is 62 whereas for silane modified $\mathrm{BaTiO}_{3}-\mathrm{PVDF}$, it is obtained as 97 . Thus modified Barium Titanate using silane gives better results compared to unmodified Barium Titanate. This is essentially associated with the increased compatibility of the silane modified Barium Titanate, with the polymeric matrices resulting in enhanced interfacial matching and reduced interfacial defects. 

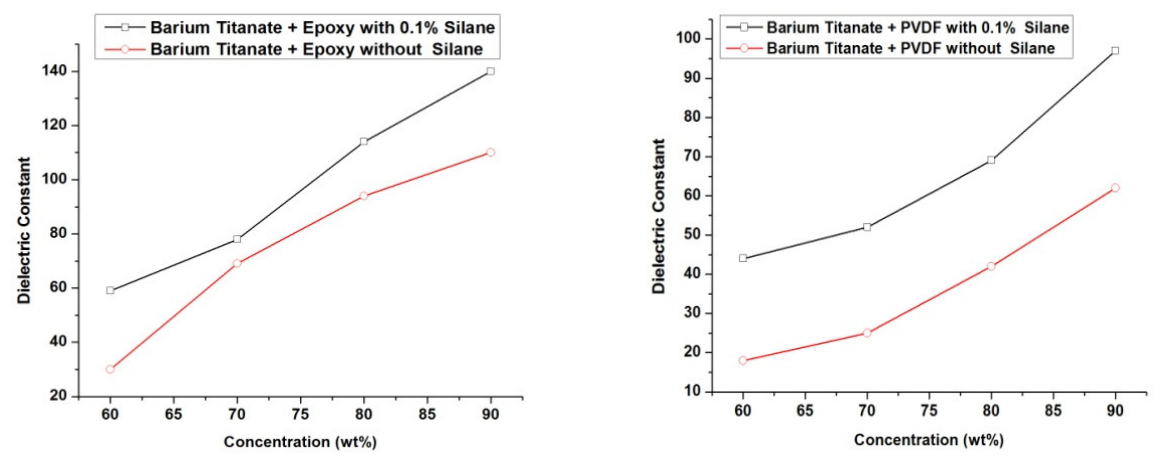

Fig. 3 Dielectric constant of with and without silane modified (a) $\mathrm{BaTiO}_{3}-$ Epoxy and (b) $\mathrm{BaTiO}_{3}-\mathrm{PVDF}$ composites at $50 \mathrm{~Hz}$ as a function of concentration (wt \%) of $\mathrm{BaTiO}_{3}$
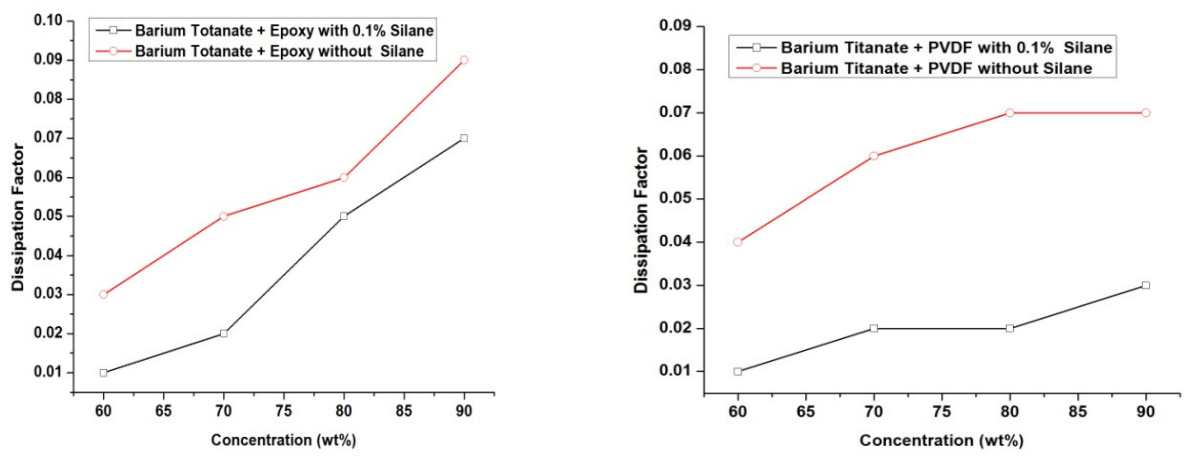

Fig. 3 Dissipation Factor of with and without silane modified (c) $\mathrm{BaTiO}_{3}-$ Epoxy and (d) $\mathrm{BaTiO}_{3}-\mathrm{PVDF}$ composites at $50 \mathrm{~Hz}$ as a function of concentration (wt \%), of $\mathrm{BaTiO}_{3}$

Figure 3 (c-d) presents plot of dissipation factor versus concentration of Barium Titanate for epoxy and PVDF polymer matrices, respectively. It is observed that dissipation factor increases with increase in the concentration of Barium Titanate for both the composite systems. Thus increase in dielectric constant correlates with an increase in dissipation factor, as the Barium Titanate concentration increased. Specifically, $90 \mathrm{wt} \%$ of the unmodified $\mathrm{BaTiO}_{3}$ - epoxy system exhibits the highest dissipation factor of 0.09 whereas the silane modified $\mathrm{BaTiO}_{3}$ - epoxy shows highest dissipation factor of 0.07 . Similarly for the unmodified $\mathrm{BaTiO}_{3}-\mathrm{PVDF}$ composites, the dissipation factor obtained is 0.07 whereas for silane modified $\mathrm{BaTiO}_{3}$-PVDF it is obtained as 0.04. In light of the importance of the dielectric constant, the above results suggest that the optimal material would be silane modified $\mathrm{BaTiO}_{3}$ at $90 \mathrm{wt} \%$ loading in the epoxy matrix. The composition has the highest dielectric constant of 140 with dissipation factor of 0.07 . 


\subsection{Temperature and Frequency dependence of dielectric properties}

The effects of frequency and temperature on dielectric constant were studied and the results are plotted in Figure 4 (a-d), on $90 \mathrm{wt} \%$ silane modified Barium Titanate in epoxy as well as PVDF matrices. Specifically Fig. 4 (a-b) presents the temperature dependence of dielectric constant and dissipation factor respectively at a frequency of $50 \mathrm{~Hz}$. It can be seen that as the temperature is increased, the ionic mechanism of polarization increases, the dielectric constant increased for epoxy as well as PVDF matrix based systems. Similarly dissipation factor increased with the increasing temperature. This change in dielectric loss is brought by an increase both in conduction of residual current and the conduction of absorption current. In Fig. 4 (c) the volume resistivity of the epoxy as well as PVDF matrix system decreases with increase in temperature. Due to heating, the atoms start vibrating and creating electrons to become carriers for current, at high temperature the resistance of Barium Titanate can fall. Similarly, Fig 4 (d) shows the frequency dependence of dielectric constant that as the frequency increases, the material's net polarization drops as each polarization mechanism ceases to contribute, and hence its dielectric constant drops as the frequency is increased for epoxy as well as PVDF matrix based systems.

Highest dielectric constant and dissipation factor are obtained as 181 and 0.25 respectively at $140{ }^{\circ} \mathrm{C}$ for epoxy-composite system, at $50 \mathrm{~Hz}$. The high dielectric constants obtained with silane modified $\mathrm{BaTiO}_{3}$-Polymer composite confirm that silane act as a molecular bridge between the polymer and the Barium Titanate filler, resulting in the formation of covalent chemical bonds across the interface, which improve the dielectric properties, at least at these low concentrations ${ }^{23,24}$. It is confirmed that silane treatment results in homogeneous microstructure with uniform distribution of high permittivity $\mathrm{BaTiO}_{3}$.

\section{Conclusion}

Based on the results obtained, the following conclusions are derived.

- Silane treatment of $\mathrm{BaTiO}_{3}$ with epoxy as well as PVDF matrices results in improved interfacial bonding and homogeneous dispersion of $\mathrm{BaTiO}_{3}$ particles in two matrices.

- At power frequency of $50 \mathrm{~Hz}$, the dielectric constant as well as dissipation factor increase monotonically, as the temperature is increased from $30^{\circ} \mathrm{C}$ to $140{ }^{\circ} \mathrm{C}$, for $90 \mathrm{wt} \% \mathrm{BaTiO}_{3}$ in epoxy as well as PVDF matrices.

- Results indicate that the dielectric constant and dissipation factor vary between $18-140$ and 0.01 to 0.09 , respectively, as the relative ratio of polymer and silane modified Barium Titanate is varied.

- The results confirm the findings of other researchers in the field that surface modification of $\mathrm{BaTiO}_{3}$ with silane at low concentration levels of order of $0.1 \%$ (of $\mathrm{BaTiO}_{3}$ ) improves particle wettability, enhances interfacial bonding, and in general improves homogeneity and dispersion of the particulate phase in epoxy as well as PVDF matrices resulting in improved dielectric properties. 


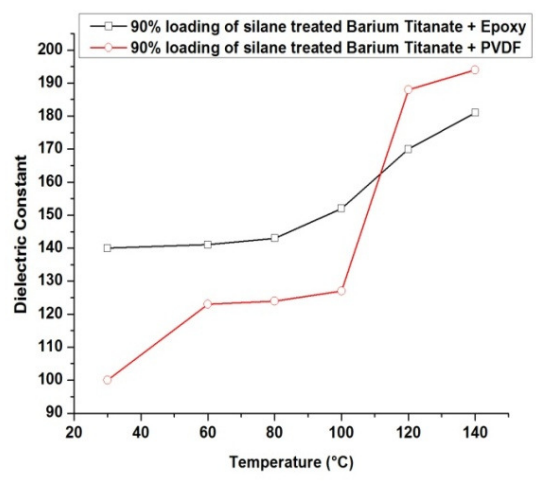

(a)

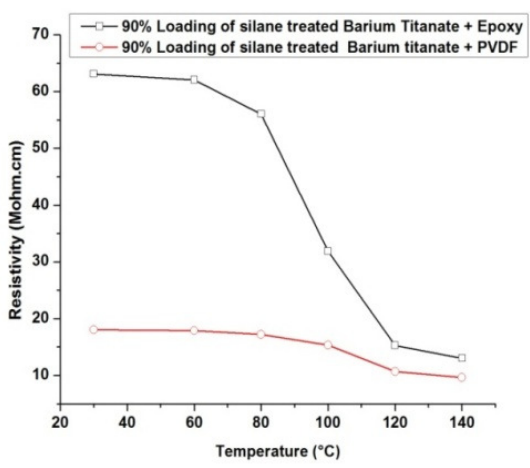

(c)

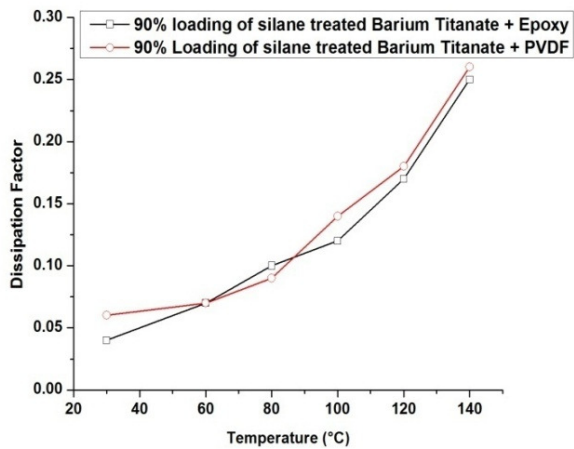

(b)

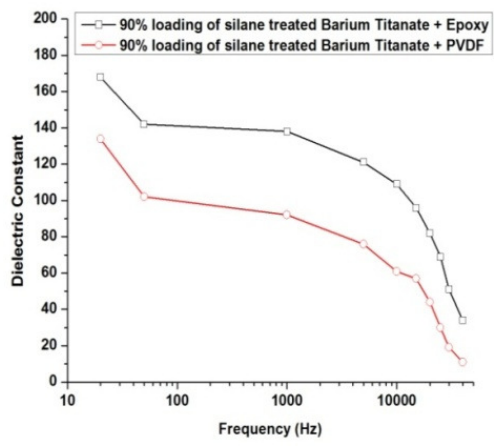

(d)

Figure 4. Effect of temperature on (a) Dielectric constant of silane modified Barium Titanate + Epoxy and Barium Titanate + PVDF (b) dissipation factor of silane modified Barium Titanate + Epoxy and Barium Titanate + PVDF (c) volume resistivity of silane modified Barium Titanate + Epoxy and Barium Titanate + PVDF (d) effect of frequency on dielectric constant of silane modified Barium Titanate + Epoxy and Barium Titanate + PVDF.

\section{Acknowledgments}

The authors are grateful to ERDA for supporting this study. The help rendered by Mr. J.P. Sheth is thankfully acknowledged.

\section{References}

1. Uchino K. and Takahashi S., Dielectric Ceramic Materials. Ceramic Transactions 100, 455468, 1999.

2. Ramajo L.A., Reboredo M.M., Castro M.S., Characterization of epoxy/BaTiO 3 composites processed by dipping for integral capacitor films (ICF), J Mater Sci., 42, 3685-3691, 2007.

3. Windlass, H., Raj, P. M., Balaraman, D., Bhattacharya, S. K. and Tummala, R. R., Colloidal processing of polymer ceramic nanocomposite integral capacitors. IEEE Trans. Electron. Pack. Manuf., 26, 100-105, 2003. 
4. Ramesh, S., Shutzberg, B. A., Huang, C. C., Gao, J. and Giannelis, E. P., Dielectric nanocomposites for integral thin film capacitors: materials design, fabrication and integration issues. IEEE Trans. Adv. Pack., 26, 17-24, 2003.

5. Rao, Y., Ogitani, S., Kohl, P. and Wong, C. P., Novel polymer-ceramic nanocomposite based on high dielectric constant epoxy formula for embedded capacitor application. J. Appl. Polym. Sci., 83, 1084-1090, 2002.

6. Bhattacharya, S. K. and Tummala, R. R., Integral passives for next generation of electronic packaging: application of epoxy/ceramic nanocomposites as integral capacitors. Microelctron. J., 32, 11-19, 2001.

7. Windlass, H., Raj, P. M., Balaraman, D., Bhattacharya, S. K. and Tummala, R. R., Polymerceramic nanocomposite capacitors for system-on-package (SOP) applications. IEEE Adv. Pack., 26, 10-16, 2003.

8. Rao, Y., Qu, J., Marinis, T. and Wong, C. P., A precise numerical prediction of effective dielectric constant for polymer-ceramic composite based on effective-medium theory. IEEE Trans. Comp. Pack. Technol., 23, 680-683, 2000.

9. Choa S.D., Lee J.Y., Hyuna J.G., Paik K.W. Study on epoxy/BaTiO 3 composite embedded capacitor films (ECFs) for organic substrate applications, Mater Sci. Eng B, 110, 233-239, 2004.

10. Dang Z.M., Fan L.Z., Shen Y., Nan C.W., Study on dielectric behavior of a three-phase $\mathrm{CF} /\left(\mathrm{PVDF}+\mathrm{BaTiO}_{3}\right)$ composite, Chem. Phys Lett., 369(1-2), 95-100, 2003.

11. Dang Z.M., Fan L.Z., Shen Y., Nan C.W., Dielectric behavior of novel three-phase MWNTs/BaTiO 3 /PVDF composites, Mater Sci. Eng B, 103(2), 140-144, 2003.

12. Bai Y., Cheng Z.Y., Bharti V., Xu H., Zhang Q.M., High-dielectric-constant ceramic-powder polymer composites, Appl Phys Lett, 76, 3804-6, 2000.

13. Herbert J.H., Ceramic dielectrics and capacitors. Gordon and Breach, New York (NY), 1985.

14. Lee H.G., Kim H.G., Ceramic particle size dependence of dielectric and piezoelectric properties of piezoelectric ceramic-polymer composites, J Appl Phys., 67, 2024-2028, 1990.

15. Zimmermann F., Voigts M., Weil C., Jakoby R., Wang P., Menesklou W., Investigation of barium strontium titanate thick films for tunable phase shifters, J Eur. Ceram Soc, 21, 20192023, 2001.

16. Dang Z.M., Wang H.Y., Xu H.P., Influence of silane coupling agent on morphology and dielectric property in $\mathrm{BaTiO}_{3} /$ Polyvinylidene fluoride composites, Appl. Phys. Lett. 89, $112902,2006$.

17. Dang Z.M., Lin Y.Q., Xu H.P., Shi C.Y., Li S.T., Bai J., Fabrication and dielectric characterization of advanced $\mathrm{BaTiO}_{3} /$ polyimide nanocomposite films with high thermal stability, Adv. Funct. Mater. 18, 1509-1517, 2008.

18. Joshi N. J., Grewal G. S., Shrinet V., Pratap A., Buch N. J., Synthesis and Characterization of Nano-Barium Titanate Prepared by Hydrothermal Process, Integrated Ferroelectrics, 115, 142-148, 2010.

19. Duran P., Gutierrez D., Tartaj J., Banares M., Moure C., On the formation of an oxycarbonate intermediate phase in the synthesis of $\mathrm{BaTiO}_{3}$ from $(\mathrm{Ba}, \mathrm{Ti})$-polymeric organic precursors., $\mathrm{J}$ Eur. Ceram Soc., 22, 797-807, 2002.

20. Duran P., Capel F., Tartaj J., Moure C., Heating-rate effect on the $\mathrm{BaTiO}_{3}$ formation by thermal decomposition of metal citrate polymeric precursors., Solid State Ionics, 141, 529539, 2001.

21. Nakamoto K., Infrared and Raman spectra of inorganic and coordination compounds. 4th ed., Wiley, New York, 1986.

22. Lenza R.F.S., Vasconcelos W.L., Synthesis of titania-silica materials by sol-gel. Mater Res., $5,497-502,2002$. 
10 A. Pratap et al.

23. Ramajo L., Castro M.S., Reboredo M.M., Effect of silane as coupling agent on the dielectric properties of $\mathrm{BaTiO}_{3}$-epoxy composites, Composites: Part A., 38, 1852-1859, 2007.

24. Iijima M., Sato N., Wuled Lenggoro I, Kamiya H., Surface modification of $\mathrm{BaTiO}_{3}$ particles by silane coupling agents in different solvents and their effect on dielectric properties of $\mathrm{BaTiO}_{3}$ /epoxy composites, Colloids and Surfaces A: Physicochem. Eng. Aspects, 352, 88-93, 2009. 